\title{
Insights on Error-Resilient Image Transmission Schemes on Wireless Network
}

\author{
Bharathi Gururaj \\ Dept of Electronics \& Communication Engg \\ ACS College of Engineering \\ Bangalore, India
}

\author{
G Sadashivappa \\ Dept. of Telecommunication Engg \\ RV College of Engg \\ Bangalore, India
}

\begin{abstract}
Usage of image as data (or signal) is quite frequent in majority of the user-centric application. However, transmission of image over non-concrete communication medium like air is still vulnerable due to inherent weakness of wireless communication e.g. interference, noise, scattering, fading, security etc. Wireless image transmission has yet unsolved problems when it comes to error resiliency. In this paper, we have reviewed the significant research contribution published in last 5 years associated with wireless image transmission, channel coding mechanism, and investigated the scale of effectiveness in the techniques based on advantages and limitations. We also extracted a significant research gap, which requires immediate attention. Hence, we propose our future direction of work with an indicative architectural design in order to address the problems identified in research gap from existing literatures. This paper is meant to brief about the existing system and practical approaches to solve such problems.
\end{abstract}

Keywords-Wireless Image Transmission: Wireless Networks; Fading; Error-Correction; Channel Coding

\section{INTRODUCTION}

Wireless communication system is gaining speed in the commercial and domestic market owing to cost-effective usage of data transfer. There are various forms of wireless communication system e.g. wireless mesh network, wireless local area network, mobile adhoc network, wireless sensor network, and all the families of IEEE 802 [1]. All of them have potential features to carry out wireless communication system but owing to wireless medium, there are various problems that affect the file transfer system. As majority of the existing communication system are designed using electrical systems, this gives rise to fluctuations of signals leading to generation of channel noise [2]. There are various forms of noise but coupled noise is quite challenging to address with interference and intermodulation talk [3]. There are different types of filters available to minimize such noise but they are not much effective in general usage [4]. The wireless communication system is used to transfer multiple forms of files out of which multimedia file systems are quite heavy and are very challenging one to be protected from integrity problems. Out of which image files are basic signal process in multimedia aspects when it comes to wireless transmission system. Various forms of disturbances implicated over wireless channels are interference, scattering, fading, noise etc. [5]. Hence, instead of deploying any filters, it is better to use channel coding mechanisms or error correction mechanisms. At present there are two types of standard error correction mechanisms forward error correction and automatic repeat request [6]. Various forms of error detection schemes are parity bits, repetitive codes, cyclic redundancy checks, hash functions, etc. All of the above are channel coding mechanisms. However, all of them are found less effective when the communication channel is a wireless sensor network or any other form of long range communication system. We find that majority of the image transmission techniques focusing on compression factor adopt the mechanism of redundancy checks from different image data. At the same time, it is highly possible that massive redundancy may be present in different image signals that are aggregated by correlated sensors. This is still an open problem. At present, there are many schemes which focus on joint source and channel coding. They are more theoretical in nature and less practical to implement. The prime reason of inapplicability of joint algorithms is that joint processing is only possible when all the chunks of image data reach a sink point from multiple sources. This process is less practical and often leads to communication overhead from the wireless nodes when data exchange occurs. We also see that studies towards wireless image transmission discuss more about the techniques of processing (transformation) towards the image and focus less on the wireless network media. Abundant number of work is already done on ODFM networks. Apart from the concept of packetization, compression, and channel coding of image transmission, another problem that arises is the algorithm testing mechanism. The efficacy of any algorithm is always proven with respect to time and space complexity and this principle is generally not seen in research manuscripts pertaining to wireless image transmission over error-prone channel. Therefore, it is only a myth that we have achieved complete success in multimedia transmission over wireless network. The significant proof of this is degraded performance of video calls in expensive smart phones over 4G or $5 \mathrm{G}$ networks even now. We believe that although there are a large number of proposals, there are no standard techniques for wireless image transmission with error resiliency features. This paper investigates the effectiveness of existing systems and presents a future work idea to overcome it. Section II discusses the existing work where different techniques are described for image transmission over wireless network followed by an explanation of the research gap in Section III. Section IV elaborates the future direction of work and Section V summarizes the paper. 


\section{EXISTING TECHNIQUES OF IMAGE TRANSMISSION}

There are various schemes presented by different researchers pertaining to image transmission over wireless communication channel. This section discusses only the recent and most frequently used techniques of research published between 2010-2016.

\section{A. Low-Density Parity Check (LDPC)}

Basically LDPC is a type of error correcting code used over wireless channel inflicted by noise. The design principle of LDPC uses bipartite graph and is found efficient mechanism to minimize the noise below a cut-off level (Shannon limit). The conventional flow of LDPC codes is shown in Fig.1
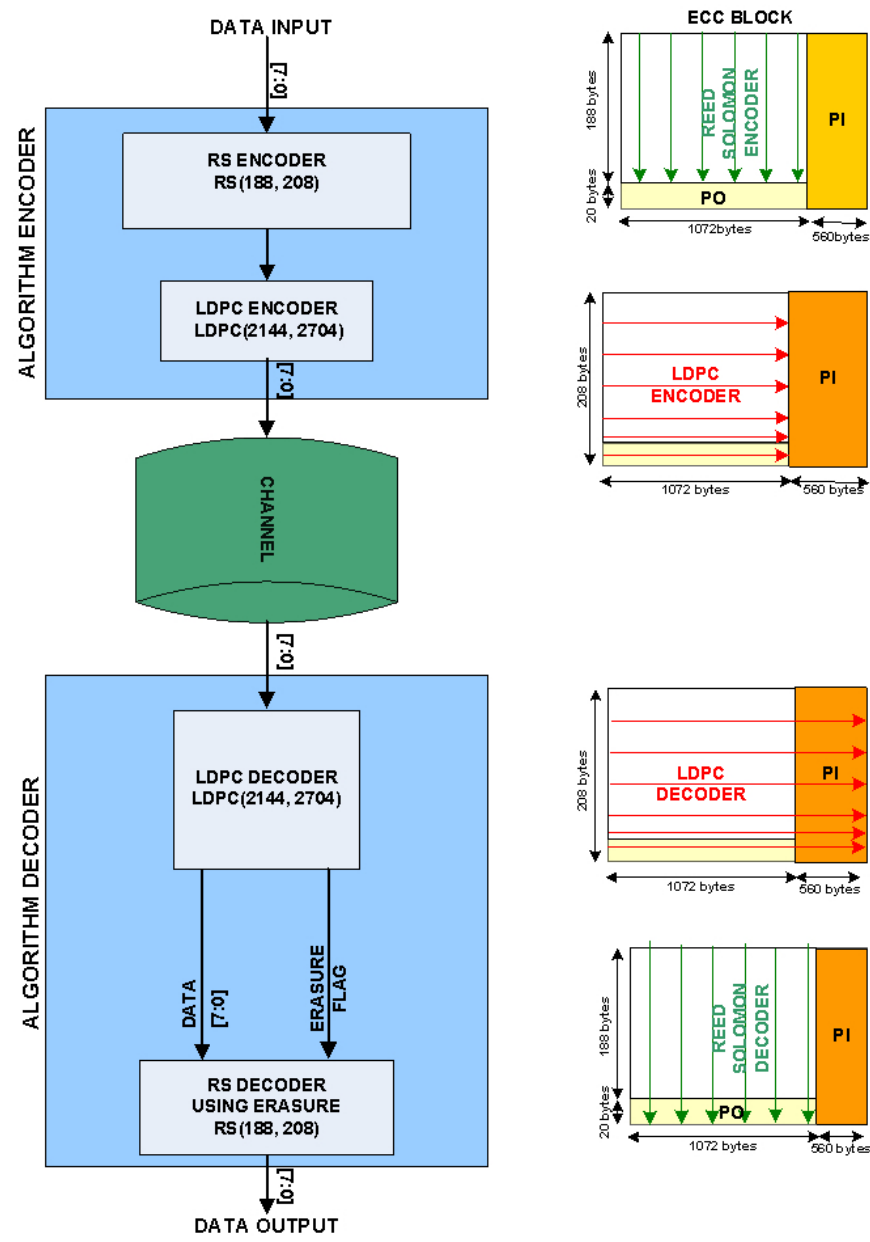

Fig. 1. Flow of Conventional LDPC Usage

Most recently, Majumder and Verma [7] adopted LDPC for progressive transmission of an image. Technique uses horizontal Reed-Solomon code along with an interleaver between it. The study outcome is testified using PSNR to find it better than existing system. A recent study by Zhang et al. [8] have used uneven LDPC codes to make the image transmission much error resistive. Considering AWGN channel, the study outcome was testified using Bit Error Rate (BER). Study by Soliman et al. [9] have also used LPDC over OFDM channel. The technique uses chaotic Baker map with a target of minimizing PAPR (Peak-average-to-power ratio). The process takes input image performs encoding with SPIHT followed by LDPC. The modulation in OFDM channel is carried out using QPSK followed by inverse Fourier operation in transmitter side. While in receiver size, chaotic de-interleaving process is carried out followed by demodulation and SPIHT decoding. Mursi et al. [10] have also used LDPC for channel coding along with a unique chaotic encryption scheme. The motive of this study was to increase the security within the communication channel for image transmission. Consideration of medical imaging and their respective transmission was also considered in prior research work. One such study was conducted by $\mathrm{Xu}$ et al. [11] by using double LDPC codes considering case study of unequal error protection. The study outcome was found to possess better PSNR performance with prior techniques. Similar technique was also continued in the research work of Wu et al. [12]. Chandrasetty and Aziz [13] have presented a technique where visual quality of the image was retained during wireless transmission. The authors have used an architecture that formulates a hierarchical matrix implemented over FPGA (Field Programmable Gate Array) in order to retain better BER performance over AWGN channel. Work carried out by Zaibi et al. [14] have considered JPEG2000 image transmission over AWGN channel. The technique uses maximum a posteriori approach for implementing the decoding scheme using arithmetic approach. The approach also uses convolution codes apart from performing an iterative decoding process. Ding and Li [15] have implemented a scheme that jointly uses both LDPC and STBC (Space Time Block Coding). The authors have used SPIHT for encoding transmission image over MIMO system BPSK modulation technique. The study outcome was tested for multiple compression rate, BER, and PSNR and found to possess better yield in comparison to conventional STBC. Mohammed et al. [16] have presented a progressive technique of image transmission using both source and channel coding approach. The technique also uses SPIHT as well as LDPC along with Lagrangian optimization technique. The input image is subjected to enhanced SPIHT along with rate optimization technique which is further subjected to LDPC encoder. The study outcome was evaluated with respect to quantity of protected bits along with usage of RS coder. The numerical outcome of the study was analyzed using MSE and PSNR. Payommai et al. [17] have introduced a technique where LDPC was used over standard Rayleigh fading channel. The objective was to enhance the data rate using LDPC code and the study outcome was witnessed with significant enhancement in BER and PSNR performance. Baldi et al. [18] have carried out a study where LDPC codes were used along with interleaver. Kasai et al. [19] have presented a study that concatenates LDPC codes with iterative codes of multiplicative origins. The study outcome was testified for lowered values of rate of frame error. Djahanshahi [20] have presented an optimization principle using LDPC codes over different types of channels. The authors have also developed multiple forms of binary image codes. Xun et al. [21] have presented a very unique study where the performance of LDPC codes during image transmission. The authors have enhanced the weighted bit flipping algorithm. The technique simultaneously performs bit flipping and enhances the bit error rate. The study outcome was shown to perform better than prior bit flipping algorithm. 


\section{B. Turbo Codes}

Turbo codes are increasingly used for error correction in image transmission over wireless medium. Usage of turbo codes are already seen in wireless communication of LTE networks as well as in satellite communication system. Fig.2 shows the turbo encode where $C_{1}$ and $C_{2}$ are two same RS encoders and $\mathrm{M}$ is memory. $\mathrm{d}_{\mathrm{k}}$ is input bits, $\mathrm{x}_{\mathrm{k}} / \mathrm{y}_{\mathrm{k}}$ are encoder outputs for $\mathrm{n}_{1}$ and $\mathrm{n}_{2}$ iterations.

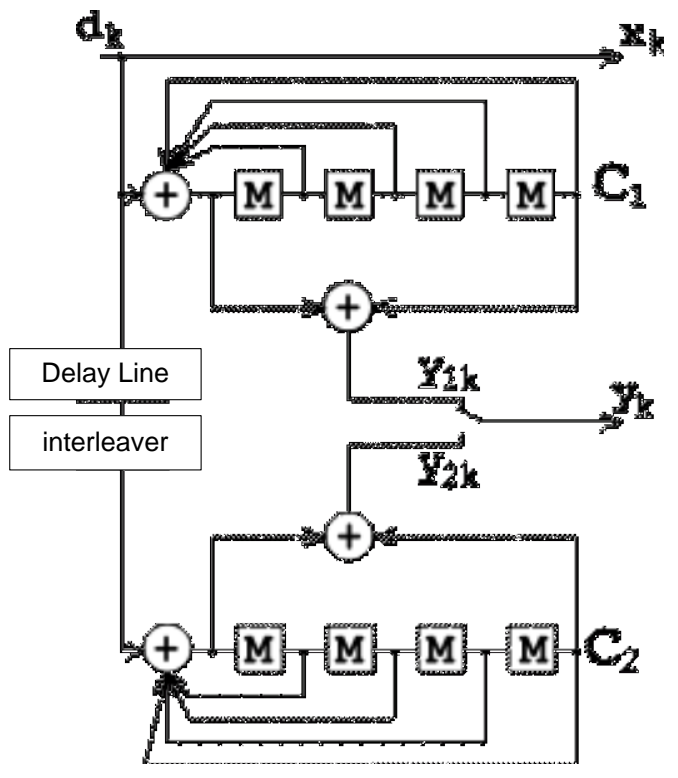

Fig. 2. Turbo Encoder

Although there are various techniques of Turbo codes adopted by researchers till date, we will only discuss the significant image transmission processes using turbo codes published between 2010-2016. The most recent implementation work of Himeur and Boukabou [22] has used turbo codes before even performing the image transmission for the purpose of minimizing the size of forwarded data. The technique has also used a noise clipping mechanism along with median filter of adaptive nature at the receiver side of OFDM system. The study outcome was evaluated with respect to transmission time, SSIM, SNR, and BER. A recent study carried out by Khalid et al. [23] have implemented multi-fold Turbo coding process in order to enhance the transmission reliability with an assistance of multiple inter leavers. The technique is capable of mitigating all unequal errors using enhanced version of trellis algorithm. The study outcome shows better Pixel-Error-Rate (PER) performance tested over OFDM channel. Aarthi et al. [24] have presented a study considering both sources coding as well as channel coding using Turbo codes. The wireless network media considered was Rayleigh fading channel and AWGN channel for image transmission. The technique uses Discrete Cosine Transform (DCT) to analyze fading and noisy channel in the form of source coding technique. The authors have enhanced the Maximum A Posteriori (MAP) which is used in turbo decoders in order to develop an error resilient image transmission scheme. Aljohani et al. [25] have introduced a source coding scheme as well as trellis coding scheme for medical image transmission. The technique uses variable length coding along with turbo trellis code on the source node while the transmission is carried out by the relay node. The study outcome shows better PSNR performance over fading channel. Problem pertaining to image quality over wireless transmission is addressed by Mao et al. [26] where a turbo code is used for unequal error protection. The technique reserves two parity bits for performing protection of higher error bits as well as lower error bits. The input images were compressed by DCT. Zhang et al. [27] have also used turbo codes for JPEG2000 image against unequal errors. The outcome was found to have better performance of BER and mean PSNR

\section{SPIHT Based Approaches}

The previous two sections have already briefed various techniques where turbo codes as well as LDPC is used along with SPIHT. Basically, Set Partitioning in Hierarchical Trees or SPIHT is used for compression purpose and its methodology is nearly equivalent to any decomposition techniques over wavelets.

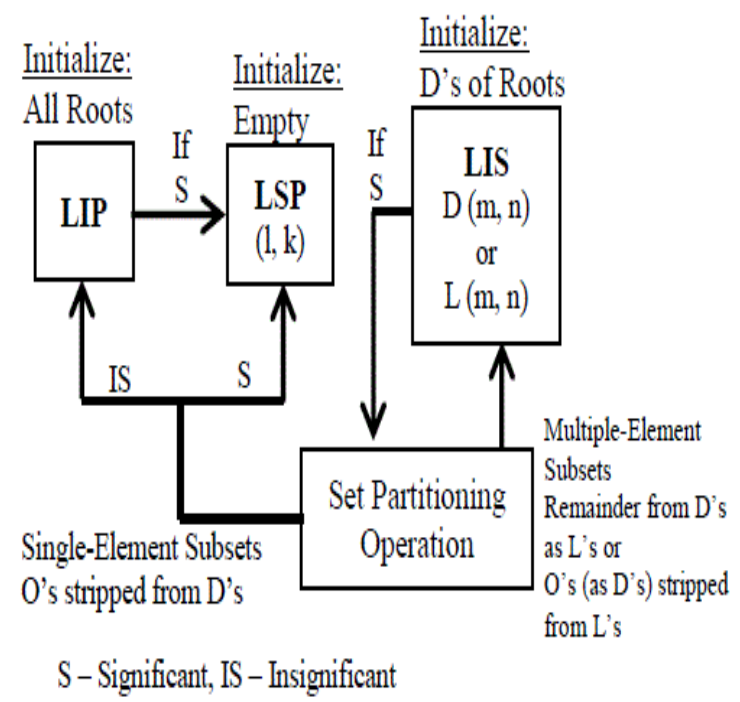

Fig. 3. Conventional SPIHT Encoding Process

Fig.3 shows the SPIHT algorithm with three different blocks i.e. LIP (List of insignificant Pixels), LSP (List of Significant Pixels), and LIS (List of insignificant Sets). The prime target of implementing SPIHT algorithm is to perform encoding reaching till anticipated bits and hence it is preferred in encoding schemes in image transmission over wireless medium.

A study carried out by Wang et al. [28] has used SPIHT algorithm along with a unique watermarking technique in order to ensure the quality of an image. The authors have used Discrete Wavelet Transform in order to decompose the signal which is further subjected to SPIHT algorithm for continuing the process of decomposition to bit planes. A unique technique of image encoding was formulated by Esmaiel and Jiang [29] where the authors have used SPIHT for transmission image over acoustic communication channel underwater. The study has also integrated use of hierarchical quadrature amplitude 
modulation scheme and Reed Solomon coding for better encoding process. The presented scheme of hierarchical quadrature amplitude modulation scheme is an enhanced version of conventional QAM modulation for making equal error protection converts to unequal error protection. Similar cadre of research work has been also carried out by Zamkotsian et al. [30]. Xiu and Zhu [31] have used SPIHT algorithm and enhanced it for transmitting image in wireless channel inflicted by noise. It has been noticed that traditional SPIHT algorithm is basically applicable to symmetrical two dimensional image block which offers less flexibility in decomposition process. Hence, Hui and Jun [32] have developed a unique unsymmetrical SPIHT encoding mechanism for eliminating the redundancies over the space blocks of image. The study outcomes show that compression ratio is better compared to conventional version of SPIHT.

\section{Channel-Based Approaches:}

From the discussion of the existing system in prior paragraphs, it is quite clear that OFDM is one of the frequently used wireless communication medium for image transmission.

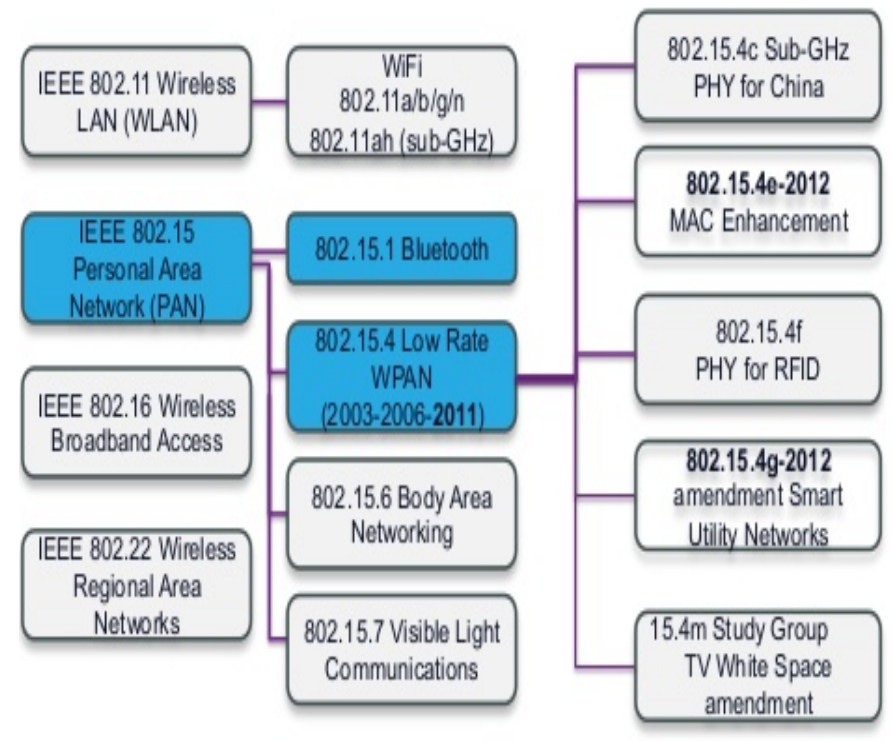

Fig. 4. IEEE Standards used for Image Transmission

But apart from OFDM, the standard family of IEEE and its variants were also experimented in the existing system (Fig.4). The work carried out by Tashiro et al. [33] presented a technique of high definition image transmission over IEEE 802.11 ac family. The technique uses both source coding and channel coding in order to accomplish lower latency and better PSNR performance.

A unique form of study was presented by Pham et al. [34] who have considered IEEE 802.15.4 for image transmission, which goes well with a wireless sensor network. El-Bendary et al. [35] have presented a similar technique but using wireless communication medium of IEEE 802.15.1 with improved rate of data over Bluetooth systems. Jelicic and Bilas [36] have carried out an investigation towards effects of image transmission over wireless network of IEEE 802.15.4/XBee. Although, the primary target of this study was for perform image transmission but the author has laid more emphasis on power minimization in such network. The technique allows minimization of power consumption using frame filling to maximum degree and restricting MAC acknowledgement. The study outcome was witnessed with $7.6 \%$ of minimization of power. There are various researchers that have used OFDM for image transmission e.g. Fatima [37], Shayegannia et al. [38], Sheikh et al. [39], Sharma et al. [40], Sharma et al. [41], Wang et al. [42], Salah [43], Tan et al. [44]. Fig.5 shows the flow of operations undertaken by OFDM methodology. 


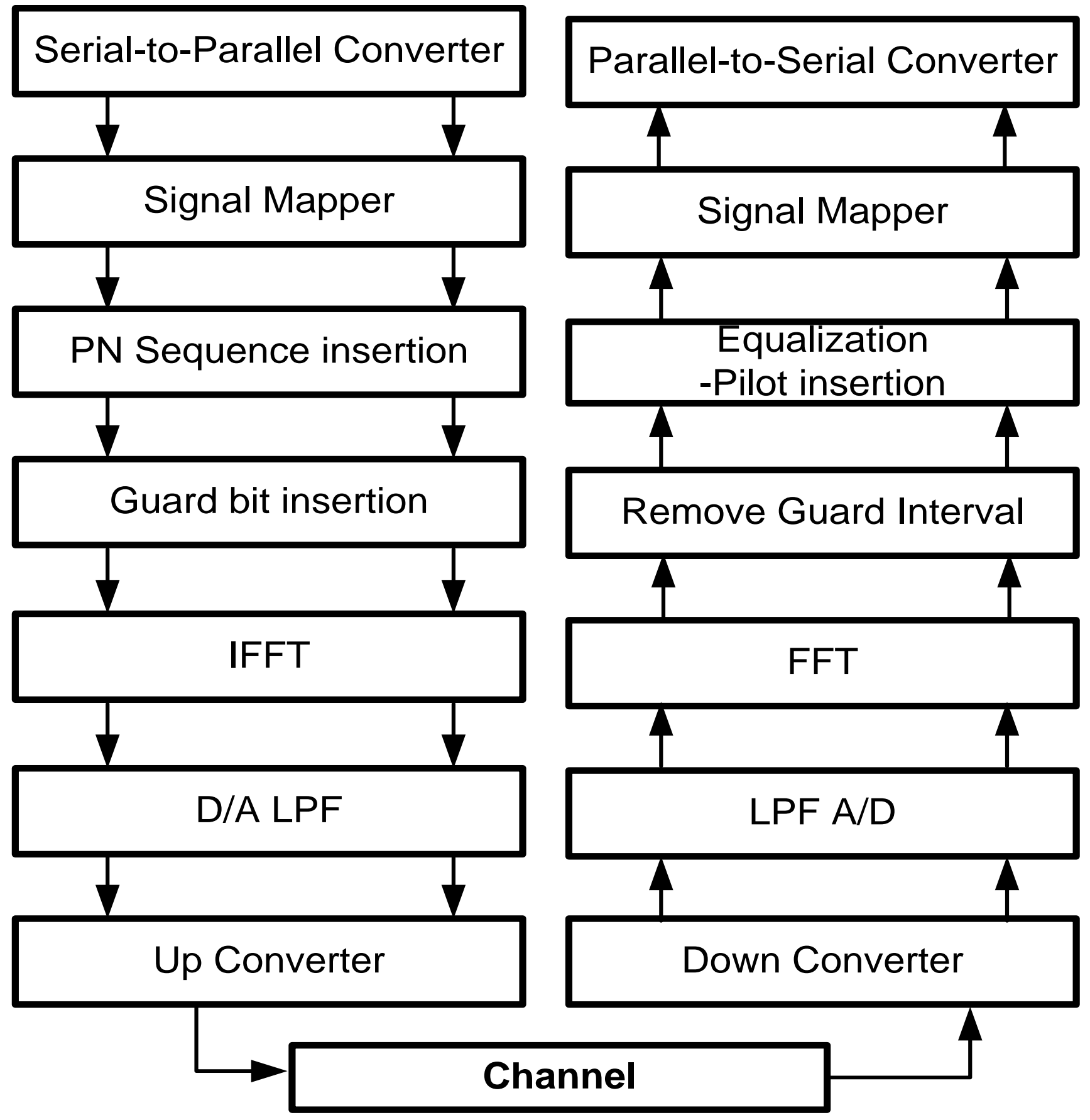

Fig. 5. OFDM Working Principle

The prime reason behind the adoption of OFDM for image transmission is its capability to mitigate interference cause due to symbols and frequency. Very often image transmission over wireless media results in loss of bits, but OFDM principle ensure recovery of maximum bits using interleaving and potential channel coding mechanism. Table 1 highlights the effectiveness of the existing system pertaining to image transmission and various techniques used. 
TABLE I. SCALING EFFECTIVENESS OF EXISTING TECHNiQUES OF IMAGE TRANSMISSION

\begin{tabular}{|c|c|c|c|c|}
\hline Authors & Problem & Technique & Advantage & Limitation \\
\hline Majumder [7] & Iterative decoding & $\begin{array}{l}\text { LDPC, Solomon Reed, } \\
\text { SPIHT }\end{array}$ & -Better PSNR outcomes & $\begin{array}{l}\text {-Tested only on natural image } \\
\text {-Less extensive analysis }\end{array}$ \\
\hline Zhang et al. [8] & $\begin{array}{l}\text { Unequal error protection } \\
\text { (AWGN Channel) }\end{array}$ & LDPC & -Better PSNR performance & $\begin{array}{l}\text {-Tested only on natural image } \\
\text {-No benchmarking }\end{array}$ \\
\hline $\begin{array}{l}\text { Soliman et al. } \\
\text { [9] }\end{array}$ & $\begin{array}{l}\text { PAPR minimization during } \\
\text { image transmission (OFDM) }\end{array}$ & SPIHT, chaotic Baker map & $\begin{array}{l}\text {-Enhances ability to be error } \\
\text { resilient }\end{array}$ & $\begin{array}{l}\text {-Tested only on natural image } \\
\text {-No benchmarking }\end{array}$ \\
\hline Mursi et al. [10] & Secure image transmission & LDPC + Chaotic theory & $\begin{array}{l}\text {-better performance of BER and } \\
\text { PSNR compared to Turbo codes }\end{array}$ & $\begin{array}{l}\text {-Tested only on natural image } \\
\text {-No benchmarking }\end{array}$ \\
\hline $\begin{array}{l}\text { Xu et al. [11], } \\
\text { Wu et al. [12] }\end{array}$ & Medical image transmission & $\begin{array}{l}\text { LDPC, Unequal error } \\
\text { protection }\end{array}$ & -Better PSNR performance & -Tested only on natural image \\
\hline $\begin{array}{l}\text { Chandrasetty } \\
{[13]}\end{array}$ & $\begin{array}{l}\text { Image quality (AWGN } \\
\text { Channel) }\end{array}$ & LDPC, FPGA & -Better BER-PSNR performance & $\begin{array}{l}\text {-Computational complexity not } \\
\text { computed. }\end{array}$ \\
\hline Zaibi et al. [14] & Source / channel coding & Arithmetic Coding, & $\begin{array}{l}\text {-Better PSNR and PER } \\
\text { performance }\end{array}$ & $\begin{array}{l}\text {-Computational complexity not } \\
\text { computed. }\end{array}$ \\
\hline Ding and Li [15] & Image transmission in MIMO & LDPC, STBC & $\begin{array}{l}\text {-better performance of BER and } \\
\text { PSNR compared to STBC }\end{array}$ & $\begin{array}{l}\text {-Computational complexity not } \\
\text { computed. }\end{array}$ \\
\hline $\begin{array}{l}\text { Mohammed et } \\
\text { al. [16] }\end{array}$ & Image transmission & LDPC, SPIHT & $\begin{array}{l}\text {-better performance of MSE and } \\
\text { PSNR }\end{array}$ & $\begin{array}{l}\text {-Computational complexity not } \\
\text { computed. } \\
\text {-Less extensive analysis }\end{array}$ \\
\hline $\begin{array}{l}\text { Payommai et al. } \\
\text { [17] }\end{array}$ & $\begin{array}{l}\text { Increasing data rate (Rayleigh } \\
\text { fading Channel) }\end{array}$ & LDPC & $\begin{array}{l}\text {-better performance of BER and } \\
\text { PSNR }\end{array}$ & $\begin{array}{l}\text {-Computational complexity not } \\
\text { computed. } \\
\text {-Less extensive analysis }\end{array}$ \\
\hline Xun et al. [18] & $\begin{array}{l}\text { Enhancing decoding } \\
\text { performance }\end{array}$ & $\begin{array}{l}\text { Enhanced weighted bit } \\
\text { flipping, LDPC }\end{array}$ & -enhanced BER performance & -narrowed scope of outcomes. \\
\hline Himeur [22] & $\begin{array}{l}\text { Image transmission over } \\
\text { OFDM channel, impulse } \\
\text { Noise }\end{array}$ & Turbo codes, median filter & -Better SNR performance & $\begin{array}{l}\text {-Computational complexity not } \\
\text { computed. }\end{array}$ \\
\hline Khalid et al. [23] & $\begin{array}{l}\text { Image transmission, noise, } \\
\text { (OFDM Channel) }\end{array}$ & Multi-fold turbo code & -better PER performance & -Less extensive analysis \\
\hline Aarthi et al. [24] & $\begin{array}{l}\text { (Rayleigh Fading + AWGN } \\
\text { channel) }\end{array}$ & $\begin{array}{l}\text { Turbo Codes, enhanced } \\
\text { MAP }\end{array}$ & -better BER performance & $\begin{array}{l}\text {-Less extensive analysis } \\
\text {-Computational complexity not } \\
\text { computed. }\end{array}$ \\
\hline $\begin{array}{l}\text { Aljohani et al. } \\
\text { [25] }\end{array}$ & $\begin{array}{l}\text { Medical image transmission } \\
\text { (Rayleigh fading) }\end{array}$ & $\begin{array}{l}\text { Variable length coding, } \\
\text { turbo trellis }\end{array}$ & -Better PSNR performance & -Less extensive analysis \\
\hline Mao et al. [26] & Quality of image & Turbo codes, DCT & -better BER performance & -Less extensive analysis \\
\hline Zhang et al. [27] & $\begin{array}{l}\text { Quality of image over } \\
\text { JPEG2000 }\end{array}$ & Turbo Codes & -better BER / PSNR performance & -Less effective benchmarking \\
\hline Wang et al. [28] & Image quality & $\begin{array}{l}\text { SPIHT, DWT, } \\
\text { watermarking }\end{array}$ & $\begin{array}{l}\text { Better accuracy, PSNR and MAE } \\
\text { performance }\end{array}$ & $\begin{array}{l}\text {-Computational complexity not } \\
\text { computed. } \\
\text {-Not tested over real-time images }\end{array}$ \\
\hline $\begin{array}{l}\text { Esmaiel and } \\
\text { Jiang [29], } \\
\text { Zamkotsian et } \\
\text { al. [30]. }\end{array}$ & Error minimization & $\begin{array}{l}\text { SPIHT, hierarchical QAM, } \\
\text { RS code }\end{array}$ & Better PSNR performance & $\begin{array}{l}\text {-Computational complexity not } \\
\text { computed. } \\
\text {-Not tested over real-time images }\end{array}$ \\
\hline $\begin{array}{l}\text { Xiu and Zhu } \\
\text { [31] }\end{array}$ & $\begin{array}{l}\text { Image transmission, noisy } \\
\text { channel }\end{array}$ & SPIHT & Better PSNR performance & $\begin{array}{l}\text {-Computational complexity not } \\
\text { computed. } \\
\text {-Not tested over real-time images }\end{array}$ \\
\hline Hui and Jun [32] & Image redundancies & Unsymmetrical SPIHT & Better compression ratio & -No test for image quality. \\
\hline $\begin{array}{l}\text { Tashiro et al. } \\
\text { [33] }\end{array}$ & $\begin{array}{l}\text { Image quality, latency } \\
\text { OFDM, MIMO }\end{array}$ & $\begin{array}{l}\text { Source coding, channel } \\
\text { coding }\end{array}$ & $\begin{array}{l}\text {-Better PSNR / BER performance } \\
\text {-Applicable to real-time image }\end{array}$ & $\begin{array}{l}\text {-Computational complexity not } \\
\text { computed. }\end{array}$ \\
\hline
\end{tabular}




\begin{tabular}{|l|l|l|l|l|}
\hline Pham et al. [34] & $\begin{array}{l}\text { Image transmission in } \\
\text { IEEE 802.15.4 }\end{array}$ & $\begin{array}{l}\text { Hardware-based approach, } \\
\text { multihop }\end{array}$ & -tested for real-time motes & $\begin{array}{l}\text {-No numerical outcome discussion. } \\
\text {-No benchmarking / complexity } \\
\text { discussion }\end{array}$ \\
\hline $\begin{array}{l}\text { El-Bendary et al. } \\
\text { [35] }\end{array}$ & $\begin{array}{l}\text { Image transmission in IEEE } \\
802.15 .1\end{array}$ & $\begin{array}{l}\text {-improved data rate, } \\
\text { Bluetooth packets }\end{array}$ & -increases throughput & -No benchmarking \\
\hline $\begin{array}{l}\text { Jelicic and Bilas } \\
\text { [36] }\end{array}$ & $\begin{array}{l}\text { Power minimization for image } \\
\text { transmission in IEEE 802.15.4 }\end{array}$ & $\begin{array}{l}\text { Restrict MAC ack, Frame } \\
\text { filling }\end{array}$ & Minimize energy & -No benchmarking \\
\hline
\end{tabular}

\section{RESEARCH GAP}

This section discusses about the significant research gap explored after reviewing the existing mechanism of wireless image transmission over error-resilient channels. Before, highlighting about the research gap, let us look into the trends of research publications till date as shown in Table 2.

TABLE II. TREND OF RESEARCH MANUSCRIPT IN IEEE XPLORE

\begin{tabular}{|c|c|c|c|c|c|}
\hline \multirow{2}{*}{ Keywords } & \multirow[b]{2}{*}{ Manuscript } & \multicolumn{2}{|c|}{ Till 2010} & \multicolumn{2}{|c|}{ After 2010} \\
\hline & & Total & Relevant & Total & relevant \\
\hline \multirow{2}{*}{$\begin{array}{l}\text { Wireless } \\
\text { image } \\
\text { transmission }\end{array}$} & Journal & 208 & 176 & 191 & 172 \\
\hline & Conference & 1280 & 1130 & 1067 & 854 \\
\hline \multirow{2}{*}{$\begin{array}{l}\text { Wireless } \\
\text { image } \\
\text { transmission, } \\
\text { OFDM }\end{array}$} & Journal & 11 & 9 & 20 & 11 \\
\hline & Conference & 81 & 74 & 158 & 121 \\
\hline \multirow{2}{*}{$\begin{array}{l}\text { IEEE 802. XX } \\
\text { family }\end{array}$} & Journal & 10 & 10 & 14 & 14 \\
\hline & Conference & 54 & 52 & 63 & 52 \\
\hline \multirow{2}{*}{ SPIHT } & Journal & 7 & 7 & 10 & 10 \\
\hline & Conference & 33 & 33 & 52 & 34 \\
\hline \multirow{2}{*}{ LDPC } & Journal & 2 & 2 & 3 & 3 \\
\hline & Conference & 22 & 22 & 46 & 41 \\
\hline \multirow{2}{*}{ Turbo codes } & Journal & 6 & 6 & 4 & 4 \\
\hline & Conference & 43 & 43 & 38 & 26 \\
\hline
\end{tabular}

The research trend shows that till date there are 3423 Journals and 2906 conference papers published. However, a closer look into the figures will show that after 2010, there are 91 less number of journals and 415 less number of conference papers published showing in the declining trend of research in this direction. This declining trend has been witnessed even though there are open issues. Therefore, the brief highlighting points of research gap pertaining to wireless image transmission schemes are as follows:

- No focus on Computational Complexity: The mechanism of wireless image transmission is increasingly used in handheld devices which uses limited computational resources and battery. Unfortunately, there is not a single study which has focused on algorithm's computational complexity, however, $2-3 \%$ of studies have focused in energy efficiency.

- Less Novelty: $80 \%$ of the studies towards energy efficiency has either used LDPC or turbo codes and the majority has already used SPIHT. Apart from there, there are less novel techniques being seen in the study.
Also, $70 \%$ of the study chooses OFDM as the wireless communication medium whereas there existing many other types of wireless networks. Studies towards image transmission using IEEE family are also quite less as compared to OFDM principle.

- Lesser focus on Spectral Correlation and allocation policies: Majority of the existing studies considers eliminating spatial correlation and extremely less on spectral correlation, which is normally seen in video sequences. Also, it has been seen that allocation of channel along with source code rate evaluation is something which is quite ignored in wireless image transmission process.

- Lack of consideration of longer communication range: At present there are many wireless communication protocols which support longer range of communication, but existing techniques have not considered such long ranges.

- Few benchmarked Studies: There is either no benchmarking or ineffective benchmarking which does not assist in highlighting best and effective mechanism to perform wireless image transmission.

\section{FUTURE RESEARCH DIRECTION}

After reviewing the existing techniques on image transmission mechanism over the wireless network, we strongly feel that there is a need of further research in this direction. This section briefly discusses the proposed line of research in order to address the research gap identified in the above study:

\section{A. Novel Technique of Stochastic-based Compression}

The prime objective of this technique is to design and develop a novel compression scheme in order to minimize the size of images with massive dimensions.

- Problem Identification: Usually images generated from Magnetic Resonance Imaging i.e. MRI are quite large and generate multiple spectrum of sequences of the image in order to assist in closer observation during diagnosis. Such images are potentially associated with both spectral and spatial correlation and the existing mechanism (i.e. DWT, DCT etc.) of transformation will be computationally significantly complex in nature especially, when such sequences of images are transmitted over wireless channel.

- Research Methodology: An analytical model can be suitably used for developing a novel transformation technique in order to eliminate spectral correlation of 
such image sequences. The technique will extract multiple bands from the images

- Anticipated Outcomes: The study outcome will evaluate the performance of compression with respect to data quality e.g. PSNR.

\section{B. Novel Error-Resilient Design of Image Transmission over wireless network}

The prime motive of this part of the study will be to design an error-resilient wireless image transmission scheme. This scheme is an enhanced version of the scheme discussed in section A.

- Problem Identification: The long ranges of wireless communication systems e.g. satellite, military communication, terrestrial microwave system has received less attention and more attention is laid on to OFDM wireless networks when it comes to wireless image transmission. The complexity of implementing packetization and channel coding differs in this case, which needs further investigation.

- Research methodology: This technique will initially build a wireless link between transmitter and receiver that will use compressive sensing for better performance. A new mechanism of the wireless channel will be designed which could operate in visible band of 390-750 nm. An encoding and decoding block will be developed consisting of encoders for compressive sensing and channel coding.

- Anticipated Outcomes: The anticipated outcome of the study will be to assess the original and reconstructed image based on signal-to-noise ratio.

\section{Novel Mathematical Scheme for error correction coding}

The core objective of this part of the study will be to develop a new error correction coding scheme that can ensure quality image transmission over error-prone wireless channel.

- Problem Identification: It is a challenging task to allocate a channel as well as rate of source code in error prone channel. The existing error correction scheme are not completely resilient against wireless network.

- Research Methodology: A novel mathematical optimization approach will be used for exploring rate allocation policies. The study will use intra coding approach and rate-distortion theory

- Anticipated Outcomes: The study outcome will be testified using PSNR, algorithm complexity,

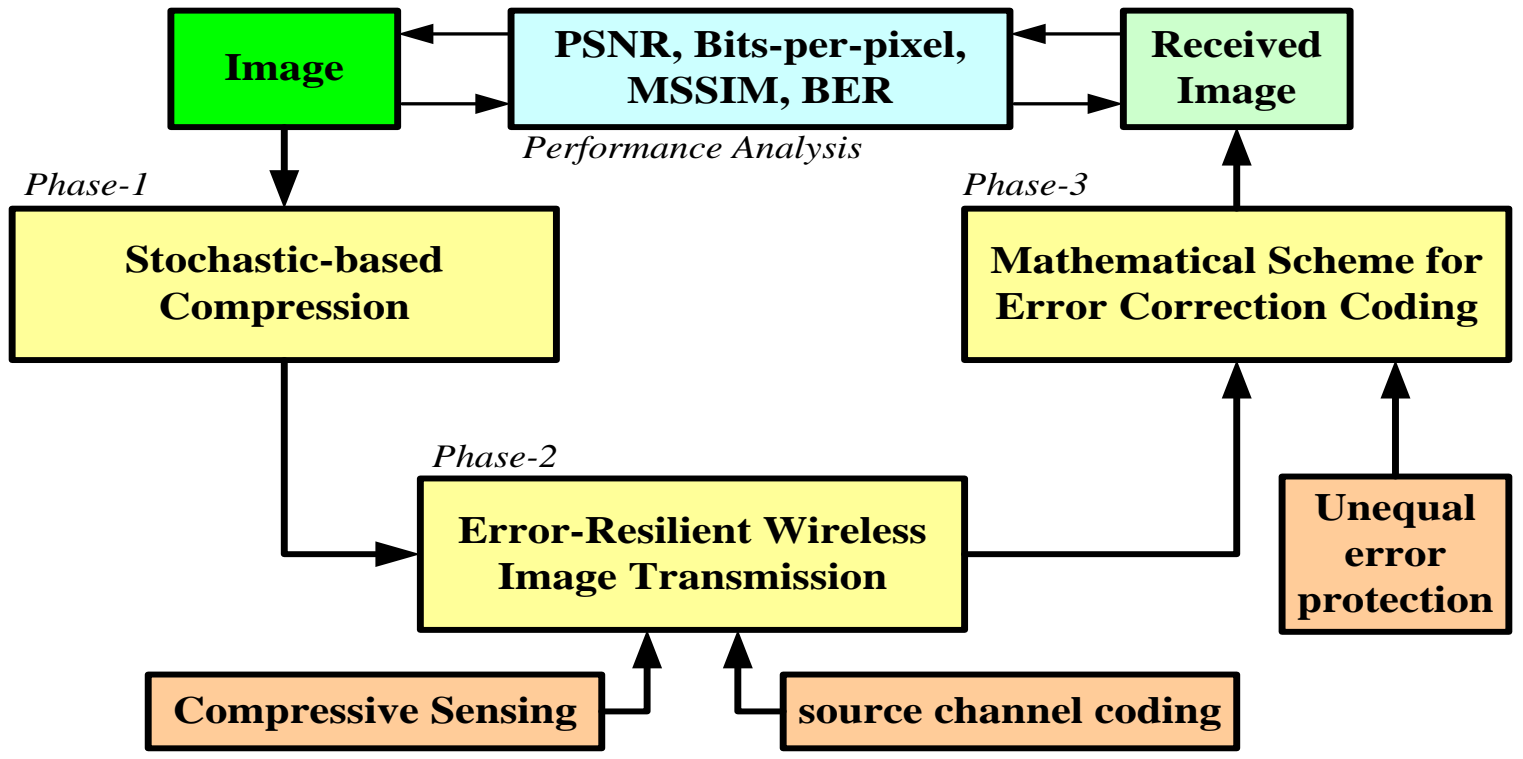

Fig. 6. Indicative Schema of Future Work Direction

\section{CONCLUSION}

An image is vulnerable to be transmitted in the wireless communication medium due to scattering, fading, interference, noise etc. Although, there has been a huge number of research work in last decade for addressing these problems, we do not have any effective or a standard algorithm to claim complete success. For an example, none of the work done using SPIHT, LDPC, turbo codes were tested for practical applicability although some of them have been tested over lab prototypes. Behaviour of wireless channel is highly dynamic in nature and calls for an extensive test environment. At present, none of the work done till date have used extensive simulation analysis or used maximum number of performance parameters other than PSNR and BER. There is no single algorithm which has been claimed to have highly reduced computational complexity as none of them has been found to be tested for time and space complexity. There is also a diminishing trend of research from 2010 onwards despite unresolved problems. We strongly believe that PSNR and BER are used to show enhanced numerical values of image but the visual quality is still not up to the mark. As most of the existing systems have been only tested over natural images and not on real-time images (which have different degrees of noise), our study will be to develop a 
framework that can offer multiple forms of integrated techniques to enhance both visual and statistical quality of an image. This framework will be used as a base for the future work direction discussed in previous section.

\section{REFERENCES}

[1] B. M. Wilamowski, J. David Irwin, Industrial Communication Systems, CRC Press, 2016

[2] P. Liu, J. Jiang, C. Wang, "Noise Analysis and Suppression for an infrared focal plane array CMOS readout circuits”, Taylor and Francis Group, Electronics and Electrical Engineering, 2015

[3] D. G. Baker, Electromagnetic Compatibility: Analysis and Case Studies in Transportation, John Wiley \& Sons, 16-Dec-2015

[4] M. S. Mahmoud, Y. Xia, Networked Filtering and Fusion in Wireless Sensor Networks, CRC Press, 2014

[5] J. West, T. Dean, J. Andrews, Network+ Guide to Networks, Cengage Learning, 2015

[6] M. M. da Silva, Cable and Wireless Networks: Theory and Practice, CRC Press, 2016

[7] S. Majumder, S. Verma, "Iterative Channel Decoding of FEC-Based Multiple Descriptions using LDPC-RS Product Codes”, International Journal of Applied Engineering Research, Vol.11, No.9, pp 6160-6167, 2016

[8] Y. Zhang, X. Li, and H. Yang, "Unequal Error Protection in Image Transmission Based on LDPC Codes”, International Journal of Signal Processing, Image Processing and Pattern Recognition, Vol.9, No.3, pp.1-10, 2016

[9] N. F. Soliman, Y. Albagory, M. A. M. Elbendary, "Chaotic Interleaving for Robust Image Transmission with LDPC Coded OFDM", Springer Journal of Wireless Personal Communication, vol.79, pp.2141-2154, 2014

[10] M. F. M. Mursi, H. Eldin H. Ahmed, "Combination of Hybrid Chaotic Encryption and LDPC for Secure Transmission of Images over Wireless Networks”, International Journal of Image, Graphics and Signal Processing, vol.12, pp.8-16, 2014

[11] L. Xu, L. Wang, S. Hong, H. Wu, "New Results on Radiography Image Transmission with Unequal Error Protection Using Protograph Double LDPC Codes”, IEEE International Symposium On Medical Information And Communication Technology, pp.1-4, 2014

[12] H. Wu, H. Jiguang, L. Xu and L. Wang, "Joint Source-Channel Coding Based on P-LDPC Codes for Radiography Images Transmission”, IEEE 11th International Conference on Trust, Security and Privacy in Computing and Communications, 2012

[13] V. A. Chandrasetty and S. M. Aziz, "Resource Efficient LDPC Decoders for Multimedia Communication" Elsevier Integration of VLSI Journal, vol.48, pp.213-220, 2015

[14] S. Zaibi, A. Zribi, R. Pyndiah, and N. Aloui, "Joint source/channel iterative arithmetic decoding with JPEG 2000 image transmission application”, Springer- EURASIP Journal on Advances in Signal Processing, vol. 114, 2012

[15] S. Ding and R. Li, "A Combined Scheme of LDPC-STBC for Image Transmission in Asynchronous Cooperative MIMO Systems", IEEE Wireless Advanced, 2012

[16] U. S. Mohammed, O. A. Omer, A. S. A. Mubark, “C29. Source-Channel Rate Allocation Scheme for Robust Progressive Image Transmission Using LDPC”, IEEE National Radio Science Conference, 2012

[17] T. Payommai, W. Chiracharit and K. Chamnongthai, "Sub-block Encoder of High-Rate LDPC Code over Fading Channel for Image Transmission”, IEEE International Conference on Electrical Engineering / Electronics, Computer, telecommunictaion, and Information technology, 2012

[18] M. Baldi, G. Cancellieri, and F. Chiaraluce, "Interleaved Product LDPC Codes”, IEEE Transactions on Communication, pp.895-901, 2011

[19] K. Kasai, D. Declercq, C. Poulliat, K. Sakaniwa, "Multiplicatively Repeated Non-Binary LDPC Codes" IEEE Transactions on Information Theory, Institute of Electrical and Electronics Engineers, vol.57, Iss.10, pp.6788-6795, 2011
[20] A. H. Djahanshahi, "Optimizing and decoding LDPC codes with graphbased techniques", Doctorial Thesis of University of California, 2010

[21] W. Z. Xun, Y. X.Qiao, W. X. Cheng, G. Dong, “An Improved IWBF Decoding Algorithm Based on LDPC Codes in the Image Transmission”, IEEE International Conference on Wireless Communication, Networking, and Information System, pp.98-101, 2010

[22] Y. Himeur, A. Boukabou, "Robust image transmission over powerline channel with impulse noise", Springer Journal of multimedia Tools applications, 2016

[23] A. Khalid, E. Khan, B. Adebisi, B. Honary, S. U. Khan, "Image transmission using unequal error protected multi-fold turbo codes over a two-user power-line binary adder channel”, IET Image Processing, 2014

[24] V. Aarthi, S.N. Kannan, N. Ramashankar, "Combined Source and Channel Coding for Image transmission using Enhanced Turbo Codes in AWGN and Rayleigh Fading Channel”, International Conference on Advanced Computing and Communication System, 2015

[25] A. J. Aljohani, H. Sun, S. X. Ng and L. Hanzo, “Joint Source and Turbo Trellis Coded Hierarchical Modulation for Context-aware Medical Image Transmission”, IEEE International Workshop on Service Science for e-Health, 2013

[26] Q. Mao, B. Xu, Y. Qin, “A New Scheme to Improve the Quality of Compressed Image Transmission by Turbo Unequal Error Protection Codes”, Seventh International Conference on Intelligent Information Hiding and Multimedia Signal Processing, 2011

[27] W. Zhang, X. Shao, M. Torki, A. H. Mohammadi, and I. V. Bajic, "Unequal Error Protection of JPEG2000 Images Using Short Block Length Turbo Codes”, IEEE Communications Letters, vol. 15, no. 6, June 2011

[28] S. Wang, D. Zheng, J. Zhao, W. J. Tam, and F. Speranza, “Adaptive Watermarking and Tree Structure Based Image Quality Estimation", IEEE Transactions On Multimedia, vol. 16, no. 2, February 2014

[29] H. Esmaiel and D. Jiang, "SPIHT Coded Image Transmission over Underwater Acoustic Channel with Unequal Error Protection using HQAM", IEEE Third International Conference on Information Science and Technology, 2013

[30] M. Zamkotsian, K. P. Peppas, G. Fovakis, F. Lazarakis, "Wireless SPIHT-encoded image transmission employing hierarchical modulation: A DSP implementation”, IEEE International Symposium on Signal processing and information Processing, 2013

[31] C. Xiu, H. Zhu, "A Modified SPIHT Algorithm Based on Wavelet Coefficient Blocks for Robust Image Transmission over Noisy Channel”, IEEE Third International Symposium on Information Science and Engineering, 2010

[32] Z. Z. Hui, Z. Jun, "Unsymmetrical SPIHT Codec and 1D SPIHT Codec", IEEE International Conference on Electrical and Control Engineering, 2010

[33] K. Tashiro, L. Lanante Jr., M. Kurosaki and H. Ochi, "High-resolution Image Transmission over MIMO-OFDM E-SDM System with JSCC", IEEE International Conference on Consumer Electronics, 2014

[34] C. Pham, V. Lecuire, J.M. Moureaux, "Performances of Multi-Hops Image Transmissions on IEEE 802.15.4 Wireless Sensor Networks for Surveillance Applications", IEEE 9th International Conference on Wireless and Mobile Computing, Networking and Communications, 2013

[35] M. A. M. El-Bendary, M. El-Tokhy, F. Shawki, and F. E. Abd-ElSamie, "Studying the Throughput Efficiency of JPEG Image Transmission over Mobile IEEE 802.15.1 Network Using EDR Packets", 6th International Conference on Sciences of Electronics, Technologies of Information and Telecommunications, 2012

[36] V. Jelicic, V. Bilas, "Reducing Power Consumption of Image Transmission over IEEE 802.15.4/ZigBee Sensor Network", IEEE International Conference on Instrumentation and Measurement technology, 2010

[37] N. Fatima, "Image Transmission over OFDM System using Trigonometric Transforms", International Conference on Communication, Information \& Computing Technology, 2015

[38] M. Shayegannia, A. Hajshirmohammadi, S. Muhaidat, "Using an Adaptive UPA Scheme with a Channel-Aware OFDM Technique for 
Wireless Transmission of JPEG2000 Images”, IEEE Canadiam Conference on Electrical and Computer Engineering, 2012

[39] L. A. Sheikh, S. A. Parah, Uzma, “Orthogonal Variable Spreading Factor (OVSF) based image Transmission using Multiple Input Multiple Output Orthogonal Frequency Division MUltiplexing (MIMO-OFDM) System”, IEEE International Conference on Communication, Devices, and Intelligent System, 2012

[40] K. Sharma, A. Mishra, A. De, "Robust Watermarked Image Transmission on OFDM Wireless Network", IEEE Canadian Conference on Electrical and Computer Engineering, 2012

[41] A. Sharma, S. De, and H. M. Gupta, "Energy-Efficient Transmission of
DWT Image over OFDM fading Channel”, IEEE International Conference on Communication Systems and Networks, 2010

[42] W-Q Wang, "Space-Time Coding MIMO-OFDM SAR for HighResolution Imaging”, IEEE transactions on geoscience and remote sensing, vol. 49, no. 8, August 2011

[43] M. M. Salah, A.A.Elrahman, "Coded OFDM scheme for image transmission over time-varying multipath rayleigh fading channels", IEEE Mediterranean Electrotechnical Conference, 2010

[44] S. S. Tan, M. J. Rim, P. C. Cosman, and L. B. Milstein, "VarianceAware Adaptive Modulation for OFDM-based Multiple Description Progressive Image Transmission”, IEEE Communications Society, 2010 